\title{
Step Test: a method for evaluating maximum oxygen consumption to determine the ability kind of work among students of medical emergencies
}

\author{
Payam Heydari ${ }^{1}$, Sakineh Varmazyar ${ }^{2}$, Ahmad Nikpey $^{3}$, Ali Safari Variani ${ }^{3}$, Mojtaba Jafarvand ${ }^{1}$
}

${ }^{1}$ M.Sc. of Occupational Health Engineering. Department Of Occupational Health Engineering, Social Determinants Health Research Center, Faculty of Health, Qazvin University of Medical Sciences, Qazvin, Iran

${ }^{2}$ Ph.D. of Occupational Health Engineering, Assistant Professor, Department Of Occupational Health Engineering, Social Determinants Health Research Center, Faculty of Health, Qazvin University of Medical Sciences, Qazvin, Iran

${ }^{3}$ Ph.D. of Occupational Health Engineering, Associate Professor, Department of Occupational Health Engineering, Qazvin University of Medical Sciences, Qazvin, Iran

Type of article: Original

\begin{abstract}
Introduction: Maximum oxygen consumption shows the maximum oxygen rate of muscle oxygenation that is acceptable in many cases, to measure the fitness between person and the desired job. Given that medical emergencies are important, and difficult jobs in emergency situations require people with high physical ability and readiness for the job, the aim of this study was to evaluate the maximum oxygen consumption, to determine the ability of work type among students of medical emergencies in Qazvin in 2016.

Methods: This study was a descriptive - analytical, and in cross-sectional type conducted among 36 volunteer students of medical emergencies in Qazvin in 2016. After necessary coordination for the implementation of the study, participants completed health questionnaires and demographic characteristics and then the participants were evaluated with step tests of American College of Sport Medicine (ACSM). Data analysis was done by SPSS version 18 and U-Mann-Whitney tests, Kruskal-Wallis and Pearson correlation coefficient.

Results: Average of maximum oxygen consumption of the participants was estimated $3.15 \pm 0.50$ liters per minute. $91.7 \%$ of medical emergencies students were selected as appropriate in terms of maximum oxygen consumption and thus had the ability to do heavy and too heavy work. Average of maximum oxygen consumption evaluated by the U-Mann-Whitney test and Kruskal-Wallis, had significant relationship with age $(\mathrm{p}<0.05)$ and weight groups $(\mathrm{p}<0.001)$. There was a significant positive correlation between maximum oxygen consumption with weight and body mass index $(\mathrm{p}<0.001)$.

Conclusion: The results of this study showed that demographic variables of weight and body mass index are the factors influencing the determination of maximum oxygen consumption, as most of the students had the ability to do heavy, and too heavy work. Therefore, people with ability to do average work are not suitable for medical emergency tasks.
\end{abstract}

Keywords: Maximum oxygen consumption, Medical emergency, Step test

\section{Introduction}

Safe work for workers, prevention of work-related illnesses, workers' participation, and performance improvement in work are four primary purposes of occupational health. Two of the strategies available to achieve these objectives are selection of workers commensurate with their jobs and periodic monitoring to quickly identify adverse effects on their health and properly prevent them. People who work in Emergency Medical Services (EMS), because of the nature of their jobs, face many dangers that may have adverse effects on their health and the health of others. Physical and physiological abilities of employees and their selection for a job based on their abilities, are necessary

\section{Corresponding author:}

Assistant Professor Dr. Sakineh Varmazyar, Department Of Occupational Health Engineering, Social Determinants Health Research Center, Faculty of Health, Qazvin University of Medical Sciences, Qazvin, Iran.

Tel: +98.2833359502, Fax: +98.2833345865, Email: Svarmazyar@qums.ac.ir

Received: April 24, 2016, Accepted: June 30, 2016, Published: March 2017

iThenticate screening: June 30, 2016, English editing: January 17, 2017, Quality control: February 12, 2017

(C) 2017 The Authors. This is an open access article under the terms of the Creative Commons Attribution-NonCommercialNoDerivs License, which permits use and distribution in any medium, provided the original work is properly cited, the use is non-commercial and no modifications or adaptations are made. 
for safe performance. The work of paramedics includes physical and psychological demands related to rescue in emergency situations and health care. Lifting and carrying patients without adequate equipment increases the risk of musculoskeletal disorders and injuries and due to musculoskeletal, cardiopulmonary, and mentally disorders, accelerates retirement. During a simulation of paramedics' tasks, an average range of heart rate was reported as 119 to 161 beats per minute and blood pressure was higher than average ( $63 \%$ of maximum heart rate) that this amount reached to a higher amount (up to $80 \%$ ) during carrying of patients (1). Therefore, due to the physical requirements of paramedics' work, a certain amount of energy is required to do the job. Therefore, in order that employees can be commensurate with the job in the terms of higher physiological conditions and more job efficiency and effectiveness, measuring the amount of power generation capacity in the profession of medical emergencies seems necessary. Maximum oxygen consumption shows the maximum oxygen rate of muscle oxygenation that is acceptable in many cases to measure the fitness between the individual and the desired job (2), and is dependent on factors such as age, sex, physical activity, heart rate, and weight (3). Therefore maximum oxygen consumption in many cases is used for measuring cardiopulmonary commensuration. Regular physical activity increases maximum oxygen consumption and subsequently increases a person's physical fitness (2). Maximum oxygen consumption is measured in direct and indirect ways. Because of high costs, required equipment, people to carry the equipment, and the need for sufficient space, direct methods of measuring maximum oxygen consumption are used for investigative and operational work. If the direct approaches are not practical, varied maximal and sub-maximal exercise tests are used to measure maximum oxygen consumption (4). The decision to use maximal or sub-maximal exercise tests depends on the required accuracy, the reasons for testing, risk level, and the availability of appropriate equipment and related people. When high accuracy is required, the maximal tests are used to determine the maximum oxygen consumption, but due to continuing the test until volitional exhaustion of participant, medical supervision and emergency equipment are needed. Therefore, in labor population, due to high risk level, sub-maximal tests are used (3-5). The purpose of sub-maximal exercise tests is determining heart rate response to one or more workloads, and using the results to predict maximum oxygen consumption. In sub-maximal exercise tests, the accuracy of estimating maximum oxygen consumption is mostly determined by heart rate responses. Parameters such as drug use, excessive intake of caffeine, high stress, illness, and high environmental temperature, influence heart rate. Usually, in order to estimate maximum oxygen consumption, exercise tests from ergometer bicycle, treadmill, and step are used. The benefits of step test are its simplicity, minimum requirement of equipment, safety, easy to carry, short rest periods, need of little practice, and the possibility to do at home. Therefore, the test should be selected based on the level of physical fitness of subjects $(3,4)$. Among research studies done in this field on firefighters, Tierney et al. (2010) mentioned that maximum oxygen consumption was obtained by maximal and sub-maximal tests 2.89 and $4.85 \mathrm{ml} / \mathrm{kg} / \mathrm{min}$ respectively (6). In addition, in the study of Vandermissen et al. (2014) the maximum oxygen consumption was reported by step test as $37.3 \mathrm{ml} / \mathrm{kg} / \mathrm{min}$ (3). Also, in the study of Davies et al. (2008), maximum oxygen consumption among male students of emergency care was estimated as $47.9 \mathrm{ml} / \mathrm{kg} / \mathrm{min}$ (7). Moreover, in other studies by Greg, Michael, Mark, and Mier maximum oxygen consumption was obtained 37 $(\mathrm{ml} / \mathrm{kg} / \mathrm{min}), 48(\mathrm{ml} / \mathrm{kg} / \mathrm{min}), 1.57(1 / \mathrm{min})$, and $48.8(\mathrm{ml} / \mathrm{kg} / \mathrm{min})$ respectively $(8-11)$. As the results of these studies show, only the maximum oxygen consumption among staff has been estimated, and few studies have examined how individuals are selected commensurate with job. Due to insufficient criteria for the selection of staff in different professions and the key role of physical capacity of emergency care staff in their ability to perform the essential duties, conducting this study seemed necessary (7). The aim of this study was to evaluate maximum oxygen consumption to determine the ability of work type among medical emergency students in Qazvin in 2016. The findings of this study can be a useful guide for the selection and employment of medical emergency staff and students to do essential duties that consequently, due to mismatch in this field, many employment problems will be solved and will have productivity of more employees.

\section{Material and Methods}

\subsection{Research Design and sampling}

This study was a descriptive - analytical, and in cross-sectional type conducted among medical emergency students in Qazvin (Iran) in 2016. The sample size was estimated by using the results of previous studies (12) with the confidence level of $95 \%$ and margin of error of $5 \%$ on 36 people. The samples were selected randomly from all male students of medical emergencies who had inclusion criteria from Qazvin University of Medical Sciences. Inclusion criteria were lack of history of cardiovascular, respiratory, and musculoskeletal diseases, avoiding the use of sedatives and hypnotics, and lack of jobs with high physical activity.

\subsection{Measurement Tool}

Physical Activity Readiness Questionnaire (PAR-Q) (4) was used including questions such as chest pain, dizziness and loss of balance, bone or joint problem, etc. in the field of general health awareness, to ensure the complete 
health of the participants. This questionnaire classifies the risk of cardiovascular disorders during exercise into three levels of low, medium, and high. If one of the participants reported one of the signs and symptoms of cardiovascular disorders, they would be placed in high risk classification and excluded from the study. The demographic questionnaire consisted of two parts. The first part was in the form of direct interview with the students about questions such as age, marital status, education level, risk of certain diseases, smoking, exercise and medication (certain medicine was not considered by the researchers). The second part includes measurable variables such as height, weight, body mass index, and pulse rate, etc. which were measured and recorded in the questionnaire by the researchers. The second part of the questionnaire measured variables such as height, weight, body mass index and pulse rate, and they too were measured and the data were recorded by researchers. Height of the participants was measured by using a tape meter with defined and standard conditions, their weight was measured by a digital scale, and their pulse rate by Beurer's rate monitor every minute, and recorded on the form. To measure maximum oxygen consumption by the step test, stairs with height of $40 \mathrm{~cm}$ were used for men so that the person who was being tested went up and down for 5 minutes and every minute 22.5 times, and then the frequency of going up and down was obtained. After the test, by using the subject's age and heart rate measured immediately after the test (beats per minute) (4) maximum oxygen consumption was calculated by the following equation (13):

$\mathrm{VO}_{2 \max }=\mathrm{AG}\left(131.5 \times \mathrm{VO}_{2}\right) /(\mathrm{HR}+\mathrm{GF}-72)$

Where:

$\mathrm{VO}_{2-\mathrm{Max}}$ : maximum oxygen consumption $(\mathrm{l} / \mathrm{min})$

HR: heart rate (beats per minute)

GF: sex factor (for men 10 and for women zero)

$\mathrm{AG}$ : age correction factor obtained by the following equation: $\mathrm{AG}=1.12-0.0073$ age. In this formula age is person's age.

$\mathrm{VO}_{2}$ (oxygen consumption) in the above formula is calculated by the following equation (5):

$\mathrm{VO}_{2}=(0.35 \times \mathrm{f})+(2.395 \times \mathrm{f} \times \mathrm{h})$

In this formula:

$\mathrm{f}$ : frequency of going up and down the steps (step per minute)

h: step height $(\mathrm{m})$

$\mathrm{Vo}_{2}$ : oxygen consumption at a steady state $(\mathrm{ml} / \mathrm{kg} / \mathrm{min})$

\subsection{Data Collection}

After justifying the subjects and before starting the work, PAR-Q questionnaire of general health and demographic characteristics questionnaire were given to participants to complete, and also height, weight, and pulse rate were measured by the above methods, and recorded. Then, while the person was wearing light clothes, exercise test of step was taken.

\subsection{Ethical Consideration}

Research Ethics Committee of Qazvin University of Medical Sciences confirmed this study with letter number of IR.QUMS.REC.1394.236. To avoid occurring physical and psychological harm to participants, following actions were taken: 1) Considering the inclusion criteria for participants; 2) Filling out the General Health Questionnaire by participants and in the case of a positive answer to a question, the participant will be excluded; 3) Monitoring the participant by doctor, in order to avoid any complications while doing the step test, 4) optional participation in the study and participants' wish to leave at any time; and 5) Confidentiality of subjects' data.

\subsection{Statistical Analyses}

Data were analyzed by using SPSS software 18 and statistical test of Pearson correlation, U-Mann-Whitney, and Kruskal-Wallis.

\section{Results}

Average of maximum oxygen consumption was estimated as $3.15 \pm 0.501 / \mathrm{min}$ by step test. Demographic characteristics of the subjects are presented in Table 1. The results of maximum oxygen consumption among medical emergencies students showed that $91.7 \%$ of students selected in terms of maximum oxygen consumption in this field, have ability to do hard and very hard work (Figure 1). The results of examining the relationship between dependent variable of maximum oxygen consumption and independent demographic variables, showed that there is a significant relationship between maximum oxygen consumption with age and weight groups, while there is no significant relationship with other dependent variables (groups of height, body mass index, exercise per week, and 
smoking) (Table 2). Pearson's correlation coefficient showed that there is a significant positive relationship between maximum oxygen consumption with weight and body mass index (Table 3 ).

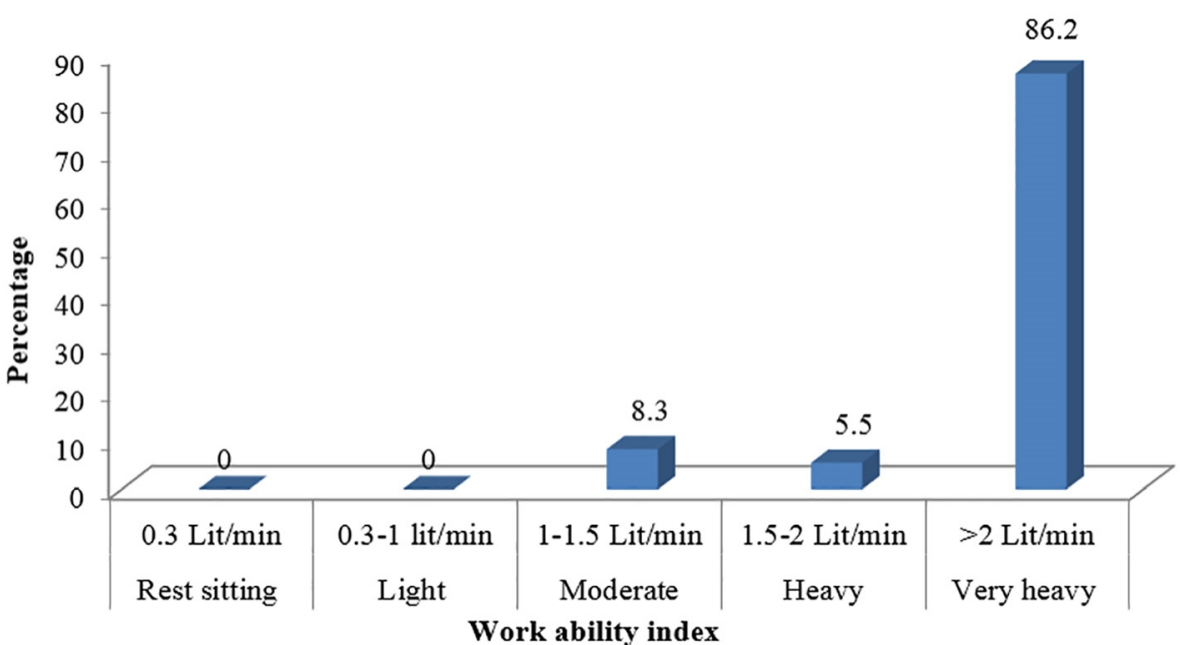

Figure 1. Percentage of work ability index based on $\mathrm{VO}_{2}$ max among subjects in the study

Table 1. Demographic data of study sample

\begin{tabular}{|c|c|c|}
\hline \multicolumn{2}{|l|}{ Variables } & $\begin{array}{l}\text { Mean } \pm \text { SD } \\
\text { Or Percent }\end{array}$ \\
\hline \multicolumn{2}{|c|}{ Age (Year) } & $21.13 \pm 1.12$ \\
\hline \multicolumn{2}{|c|}{ Weight $(\mathrm{Kg})$} & $70.05 \pm 9.61$ \\
\hline \multicolumn{2}{|c|}{ Height $(\mathrm{Cm})$} & $177.77 \pm 5.72$ \\
\hline \multicolumn{2}{|c|}{ BMI $\left(\mathrm{Kg} / \mathrm{m}^{2}\right)$} & $22.13 \pm 2.56$ \\
\hline \multicolumn{2}{|c|}{ Exercise in week (Hour) } & $6.37 \pm 5.36$ \\
\hline \multirow[t]{2}{*}{ Smoking } & 11.1 & 11.1 \\
\hline & 88.9 & 88.9 \\
\hline
\end{tabular}

Table 2. The relationship of population characteristics with $\mathrm{VO}_{2}$ max based on Mann-Whitney or Kruskal-Wallis tests

\begin{tabular}{|c|c|c|c|c|c|}
\hline \multicolumn{2}{|l|}{ Variables } & \multirow[t]{2}{*}{$\mathrm{n}(\%)$} & $\mathrm{VO}_{2 \max }$ & \multirow[t]{2}{*}{$\mathrm{Z}$ or $\mathrm{k}^{2}$} & \multirow[t]{2}{*}{ p-value } \\
\hline \multirow{3}{*}{ Age Group (Year) } & & & Mean \pm SD & & \\
\hline & $19-21$ & $22(61.1)$ & $3 \pm 0.53$ & \multirow[t]{2}{*}{-2.012} & \multirow[t]{2}{*}{$0.045^{*}$} \\
\hline & $22-24$ & $14(38.9)$ & $3.40 \pm 0.39$ & & \\
\hline \multirow[t]{4}{*}{ Weight Group (Kg) } & $<60$ & $6(16.7)$ & $2.60 \pm 0.42$ & \multirow[t]{4}{*}{19.128} & \multirow[t]{4}{*}{$0.000^{* *}$} \\
\hline & $60-70$ & $15(41.7)$ & $3 \pm 0.36$ & & \\
\hline & $71-80$ & $9(25.0)$ & $3.60 \pm 0.31$ & & \\
\hline & $>80$ & $6(16.7)$ & $3.40 \pm 0.31$ & & \\
\hline \multirow[t]{3}{*}{ Height Group (Cm) } & $<170$ & $5(13.9)$ & $3 \pm 0.41$ & \multirow[t]{3}{*}{1.104} & \multirow[t]{3}{*}{0.576} \\
\hline & $170-180$ & $20(55.6)$ & $3.13 \pm 0.57$ & & \\
\hline & $>180$ & $11(30.6)$ & $3.27 \pm 0.42$ & & \\
\hline \multirow[t]{4}{*}{ BMI } & $<18.5$ & $2(5.6)$ & $2.37 \pm 0.42$ & \multirow[t]{4}{*}{4.403} & \multirow[t]{4}{*}{0.116} \\
\hline & $18.5-24.99$ & $32(88.9)$ & $3.18 \pm 0.48$ & & \\
\hline & $25-29.99$ & $2(5.6)$ & $3.43 \pm 0.40$ & & \\
\hline & $>30$ & $0(0.0)$ & - & & \\
\hline \multirow[t]{4}{*}{ Exercise in week (Hour) } & $0-3.99$ & $12(33.3)$ & $3.16 \pm 0.58$ & \multirow[t]{4}{*}{0.901} & \multirow[t]{4}{*}{0.825} \\
\hline & $4-7.99$ & $15(41.7)$ & $3.18 \pm 0.58$ & & \\
\hline & $8-11.99$ & $5(13.9)$ & $3 \pm 0.45$ & & \\
\hline & $12-15$ & $4(11.1)$ & $3.31 \pm 0.18$ & & \\
\hline \multirow[t]{2}{*}{ Smoking } & No & $32(88.9)$ & $3.16 \pm 0.50$ & \multirow[t]{2}{*}{-0.503} & \multirow[t]{2}{*}{0.645} \\
\hline & Yes & $4(11.1)$ & $3.06 \pm 0.21$ & & \\
\hline
\end{tabular}

Z: U- Mann-Whitney test output; $\mathrm{K}^{2}$ : Kruskal-Wallis test output; *Significant in level $\mathrm{P}<0.05$ 
Table 3. The result of the correlation coefficient between population variables and $\mathrm{VO}_{2}$ max

\begin{tabular}{|l|l|l|}
\hline Variables & Pearson correlation(r) & p-value \\
\hline Age $($ Year $)$ & 0.30 & 0.07 \\
\hline Weight $(\mathrm{Kg})$ & 0.61 & ${ }^{*} 0.001$ \\
\hline Height $(\mathrm{Cm})$ & 0.30 & 0.06 \\
\hline BMI $\left(\mathrm{Kg} / \mathrm{m}^{2}\right)$ & 0.55 & ${ }^{*} 0.001$ \\
\hline Exercise in week (Hour) & -0.32 & 0.85 \\
\hline
\end{tabular}

*Significant in level $\mathrm{P}<0.01$

\section{Discussion}

Medical emergencies are jobs in emergency situations that, because of the nature of the work, require high physical ability and fitness to perform tasks in difficult conditions. In the present study, the mean and standard deviation of maximum oxygen consumption were obtained $3.15 \pm 0.50(1 / \mathrm{min})$ by step test among students of medical emergencies, and this amount is approximately close to the results of studies conducted on firefighters by Tierney et al. (4.85 1/min), Vandermissen et al. (37.3 $\mathrm{ml} / \mathrm{kg} / \mathrm{min})$, and Farhadi et al. $(3.65 \mathrm{l} / \mathrm{min})$. Mier et al. obtained 48.8 $\mathrm{ml} / \mathrm{kg} / \mathrm{min}$ in the studied sample and DeRisi et al. $3.21 \mathrm{l} / \mathrm{min}$, and also in maximum oxygen consumption had approximately similar results. Given that climatic conditions of study place affect maximum oxygen consumption; lack of the situation control could be a contributing factor to having different amounts in different studies $(3,6,11$, 14-16). This study showed a significant relationship between maximum oxygen consumption and age groups of studied samples. The findings are consistent with the results of the study by Daneshmandi et al. (17) but are not consistent with other studies (12, 15, 18-20). In some studies, such as Betik and Hepple (21), it was concluded that oxygen consumption will be decreased as you grow older, and the reason is based on decrease of maximum heart rate in older age. As you grow older, maximum heart rate will be decreased and this is an important factor in reducing oxygen consumption, the findings are not consistent with the results of the present study. The significant relationship between age groups are variable, and maximum oxygen consumption in this study may be related to the point that most of the selected people were in the age range of 19-24. Therefore, the relationship between maximum oxygen consumption and age groups is due to young age range of the studied people. Pearson correlation test showed a positive and significant relationship between maximum oxygen consumption and weight and body mass index. In other words, in this study, by increase of weight and body mass index, maximum oxygen consumption has been increased. The study conducted by Atomi et al., on two groups of lean and obese women, revealed that weight is an influencing factor in maximum oxygen consumption (22). This could be the result of expanding cardiopulmonary and musculoskeletal systems and consequently, increasing absorption of oxygen and glucose, or more food delivery to muscles through the bloodstream. In this case, the muscle will is to do more mechanical work by spending more energy (18). The findings of studies conducted by Heydari et al. (18), DeRisi et al. (23), Nazem et al. (24), and Choobineh et al. (19), confirm the findings of this research. However in other studies, weight variable has had no effect on increase or decrease of maximum oxygen consumption in individuals (17). But the findings of studies conducted by Farhadi et al. (15) and Rafiee-Pour et al. (25) showed that the amount of maximum oxygen consumption will be decreased by the increase of weight and body mass. In the studies of Gaeini et al. and Grassi et al. there was a significant negative correlation between BMI index and oxygen consumption. In other words, by increasing BMI index, oxygen consumption was decreased significantly $(23,26)$. According to Table 2 , it can be stated that there is no significant relationship between maximum oxygen consumption and smoking that this finding is not consistent with the results of studies conducted by Choobineh et al. (19) and Habibi et al. (27). The average of maximum oxygen consumption in smokers is less than nonsmokers and one reason for this may be blood saturation of carbon monoxide $(\mathrm{CO})$ existing in cigarette smoke, which subsequently decreases oxygen carrying capacity of blood (28). Also, due to deposition of nicotine existing in cigarettes into the inner lining of arteries, and decreasing oxygen, absorption capacity in muscles reduces the oxygen consumption in smokers (16). Lack of relationship between maximum oxygen consumption and smoking in this study may be due to the remarkable low number of smokers compared to nonsmokers. This imbalance makes smoking effectless on maximum oxygen consumption, and the studies conducted by Hosseinabadi et al. (29) and Rafiee-Pour et al. (25) have confirmed the lack of relationship. It should be noted that the insufficient number of samples in the present study, may be a factor of inconsistency between these findings and findings of other studies. Therefore, it is suggested that further studies on more non-uniform populations are done, in terms of smoking, to obtain more definitive results about the effects of smoking on oxygen consumption.

The results of the present study, showed that there is no significant relationship between maximum oxygen consumption and hours of exercise per week. The reason may be more exercise of studied subjects during the week. 
This finding is consistent with the results of studies conducted by Farhadi et al. (15), Matlabi et al. (16), and DeRisi et al. (23) but is not consistent with other studies $(19,27)$. By increasing the hours of exercise per week, oxygen consumption will be increased and in justification of this issue, can be said that physical exercise makes heart muscles stronger and thus increases stroke volume and a person's aerobic capacity (30).

\section{Study limitations}

Among the limitations of this study, we can mention the selection of medical emergencies personnel, merely based on maximum oxygen consumption and lack of considering other aspects of physical fitness such as health-related physical fitness (cardiovascular endurance, body composition, muscular endurance, muscular strength, flexibility) and skill-related physical fitness (speed, power, balance, agility, reaction time, and coordination), because one person may be determined appropriate and selected for medical emergencies job, in terms of oxygen consumption, but he or she has weakness in others aspects of physical fitness. Therefore, wrong selection of people merely based on one aspect of fitness, may endanger a person's health in the long term.

\section{Conclusions}

The results showed that maximum oxygen consumption has no significant relationship with the groups of height, body mass index, hours of exercise per week, and smoking, but there is a significant relationship between maximum oxygen consumption and age and weight group of medical emergency students. With regards that medical emergency is among the most important and hard jobs, it requires people with high ability and physical fitness for the job. According to the results, $91.7 \%$ of those surveyed in terms of oxygen consumption, have been selected appropriately for medical emergency jobs. Therefore, in order to select people more accurately for medical emergency jobs, it is recommended performing oxygen consumption tests and selecting those whose test results are in the range of ability to do hard and very hard work. In the case of wrongly selecting people to enter the job, appropriate training programs can be used to increase people's oxygen consumption.

\section{Acknowledgments:}

This research is the result of a thesis for Master of Science with the title of determining maximum oxygen consumption in medical emergency jobs, and using the results to select students of this field. The authors thank and appreciate Qazvin University of Medical Sciences for funding the project as well as Chia Hakimi, Sajad Ataei, and Elnaz Mohammadzadeh for their cooperation in data collection.

\section{Conflict of Interest:}

There is no conflict of interest to be declared.

\section{Authors' contributions:}

All authors contributed to this project and article equally. All authors read and approved the final manuscript.

\section{References:}

1) Waldemer W, Karwowski M. Interventions, Controls, and Applications in Occupational Ergonomics. 2 ed 2006. 374-90.

2) Siconolfi SF, Garber CE, Lasater TM, Carleton RA. A simple, valid step test for estimating maximal oxygen uptake in epidemiologic studies. Am J Epidemiol. 1985; 121(3): 382-90. PMID: 4014128.

3) Vandersmissen G, Verhoogen R, Van Cauwenbergh A, Godderis L. Determinants of maximal oxygen uptake (VO $2 \mathrm{max}$ ) in fire fighter testing. Applied ergonomics. 2014; 45(4): 1063-6. doi: 10.1016/j.apergo.2014.01.001.

4) Williams L, Wilkins. ACSM's Guidelines for Exercise Testing and Prescription. 9 ed: American College of Sport Medicine. 2013; 73-7.

5) Group TF. International Encyclopedia of Ergonomics and Human Factors. 2 ed: CRC Press. 2006.

6) Tierney MT, Lenar D, Stanforth PR, Craig JN, Farrar RP. Prediction of aerobic capacity in firefighters using submaximal treadmill and stairmill protocols. J Strength Cond Res. 2010; 24(3): 757-64. doi: 10.1519/JSC.0b013e3181c7c282. PMID: 20145563.

7) Davies S, Naidoo N, Parr B. Physical performance characteristics of South African male and female Emergency Care students (ECS). Ergonomics SA. 2008; 20(2): 3.

8) Kline GM, Porcari JP, Hintermeister R, Freedson PS, Ward A, McCarron RF, et al. Estimation of VO2max from a one-mile track walk, gender, age, and body weight. Med Sci Sports Exerc. 1987; 19(3): 253-9. doi: 10.1249/00005768-198706000-00012. PMID: 3600239. 
9) Morris M, Lamb KL, Hayton J, Cotterrell D, Buckley J. The validity and reliability of predicting maximal oxygen uptake from a treadmill-based sub-maximal perceptually regulated exercise test. Eur J Appl Physiol. 2010; 109(5): 983-8. doi: 10.1007/s00421-010-1439-1. PMID: 20352257.

10) Loftin M, Sothern M, Warren B, Udall J. Comparison of VO2 peak during treadmill and cycle ergometry in severely overweight youth. J Sports Sci Med. 2004; 3(4): 254-60. PMID: 24624011, PMCID: PMC3938064.

11) Mier CM, Gibson AL. Evaluation of a treadmill test for predicting the aerobic capacity of firefighters. Occup Med (Lond). 2004; 54(6): 373-8. doi: 10.1093/occmed/kqh008. PMID: 15347781.

12) Firoozeh M, Saremi M, Maleki A, Kavousi A. Investigation into Maximal Aerobic Capacity and its Associated Factors in Firefighters. Iran Occupational Health. 2015; 12(3): 15-26.

13) Tayyari F, Smith J. Occupational Ergonomics Principles and Applications. Illustrated ed. R. Parsaei H, editor: Springer US. 1997.

14) Zare Derisi F, Rastegar L, Hosseini S, Daneshmandi H, Choobineh A, Mohammadbeigi A. Correlation of Astrand and ACSM Protocols in Estimating the Maximum Aerobic Capacity (Vo2-Max). Journal of Ergonomics. 2014; 1(3): 27-35.

15) Farhadi S, Abazari M, Babayi Y. Design and fabrication of adjustable stair stepping test and its use for measurement of the maximum aerobic capacity in fire fighters. 1st Biennial Iranian Conference on Ergonomics; Hamedan University of Medical Sciences 2014; 1-11.

16) Matlabi Kashani M, Lahmi M. Evaluation of physical work capacity at machining process industry in tehran. Journal Elam of Medical Sciences. 2000; 9(26-27): 21-8.

17) Daneshmandi H, Choobineh A, Rajaei Fard A. Estimation of aerobic capacity and determination of its associated factors among male workers of industrial sector of Shiraz city, 2010. Iran Occupational Health. $2011 ; 8(3): 48-58$.

18) Heydari P, Varmazyar S, Mohammadzadeh E. Factors affecting estimation of the maximum aerobic capacity by treadmill test in students of medical emergencies in Qazvin. The Journal of Qazvin University of Medical Sciences. 2016; 19(6): 65-72.

19) Choobineh A, Barzideh M, Gholami T, Amiri R, Tabatabaei HR, Almasi Hashyanie A. Estimation of aerobic capacity (Vo2-max) and study of its associated factors among male workers of industrial factories in Sepidan/Fars province, 2009. Jundishapur scientific medical Journal. 2011; 10(1(70)): 1-12.

20) Chatterjee S, Mitra SK, Samanta A. Aerobic capacity of the brick-field workers in eastern India. Ind Health. 1994; 32(2): 79-84. doi: 10.2486/indhealth.32.79. PMID: 7806448.

21) Betik AC, Hepple RT. Determinants of VO2 max decline with aging: an integrated perspective. Appl Physiol Nutr Metab. 2008; 33(1): 130-4. doi: 10.1139/H07-174. PMID: 18347663.

22) Atomi Y, Miyashita M. Maximal oxygen uptake of obese middle-aged women related to body composition and total body potassium. The Journal of sports medicine and physical fitness. 1984; 24(3): 212-8. PMID: 6527517.

23) Grassi GP, Turci M, Sforza C. Aerobic fitness and somatic growth in adolescents: a cross sectional investigation in a high school context. J Sports Med Phys Fitness. 2006; 46(3): 412-8. PMID: 16998445.

24) Nazem F, Jalili M, Farahpour N, Heydarianpour A. Predictive model practical capacity through daily step count in men 65-40 years old with an active lifestyle. Sport Biosciences (move). 2012; (11): 55-73.

25) Rafieepour A, Farasati F, Kalantari S, Motamedzadeh M, Rafieepour E. The estimated maximum aerobic capacity and individual habits and demographic factors that, in the University of Medical Sciences. Journal of Qom University of Medical Sciences. 2014; 8(3): 33-40.

26) Gaeini A, Rahmaninia F, Hosseini M. The relationship between aerobic power, anthropomentric measurement and body composition of non-athlete females students. Olympic. 2000; 8(3-4): 57-66.

27) Habibi EA, Moghiseh M, Khoshzat N, Taheri MR, Gholamian J, Khoshhali M. Estimate aerobic capacity and determine the level of physical activity students. Journal of Research Health System. 2013; 9(9): 951-9.

28) Klausen K, Andersen C, Nandrup S. Acute effects of cigarette smoking and inhalation of carbon monoxide during maximal exercise. Eur J Appl Physiol Occup Physiol. 1983; 51(3): 371-9. doi: 10.1007/BF00429074. PMID: 6685036.

29) Hosseinabadi S, Hamidi Bagejan P, Ebrahimian H, Barkhordari A, Bandpe TR. Estimate aerobic capacity and physical work capacity of workers. Journal Knowledge \& Health. 2013; 8(3): 131-7.

30) Hall G. Medical physiology. 12, editor. Tehran: Arjomand. 2011; 1326. 